\title{
Ensuring the best care to cancer patients in the COVID-19 pandemic era: insights and challenges.
}

\author{
Paolo Baldo ${ }^{1}$, Sara $\mathrm{Cecco}^{2}$, Diana Crestan ${ }^{2}$, Sara Francescon$^{2}$, Elisa Giacomin ${ }^{2}$, Liana \\ Dalmas $^{2}$, Paola Molin ${ }^{3}$, and Fabio Puglisi ${ }^{2}$ \\ ${ }^{1}$ CRO Aviano Centro Riferimento Oncologico \\ ${ }^{2}$ Centro di Riferimento Oncologico \\ ${ }^{3}$ University of Udine
}

May 18, 2020

\begin{abstract}
On 11/3/2020, the World Health Organization declared that COVID-19 is a pandemic emergency. In this scenario, cancer patients are to be considered at higher risk, being fragile and often with disease-related or treatment-induced immunodepression. Furthermore, this health emergency generates a harmful psychological impact on these patients, who fear a lot about losing the continuity of cancer therapy. This fact must be carefully considered by health personnel, especially in countries where heavy risk containment and social distancing measures, the only epidemiological strategies currently considered adequate, are in force. In short, this is a very delicate challenge for health professionals engaged in oncology. Here, we report some insights and challenges that healthcare professionals face daily in caring for cancer patients.
\end{abstract}

Title of the article:

Ensuring the best care to cancer patients in the COVID-19 pandemic era: insights and challenges.

Type of paper: Brief Reports

Keywords: COVID-19; cancer, anti-infective agents; psychological, adaptation; risk-reduction behavior; epidemiology, comorbidity.

\section{Author list:}

Paolo Baldo ${ }^{1}$, Sara Cecco $^{1}$, Elisa Giacomin ${ }^{1}$, Sara Francescon ${ }^{1}$, Liana Dal Mas ${ }^{1}$, Diana Crestan $^{1}$ Paola Molin ${ }^{3}$ and Fabio Puglisi ${ }^{2}$

1. Hospital Pharmacy Unit, Centro di Riferimento Oncologico Aviano (CRO) - IRCCS, Italy.

2. Department of Medicine, University of Udine; Department of Medical Oncology, Centro di Riferimento Oncologico Aviano (CRO) - IRCCS, Italy.

3. Child Neuropsychiatric Unit "Bassa Friulana", Udine University Health Service (ASUFC), Italy.

Paolo Baldo ${ }^{1}$, corresponding author, Director of Hospital Pharmacy Unit, Centro di Riferimento Oncologico Aviano (CRO) - IRCCS, Italy. Research support: concept, writing, team coordination, submission

Sara Cecco, Hospital Pharmacy Unit, IMS Investigational Medicines Service, di Riferimento Oncologico Aviano (CRO) - IRCCS, Italy Research support: writing, search on the web and oncology websites 
Diana Crestan, Hospital Pharmacy Unit, IMS Investigational Medicines Service, di Riferimento Oncologico Aviano (CRO) - IRCCS, Italy Research support: writing, search on the web for experimental drugs in clinical trials

Sara Francescon, Hospital Pharmacy Unit, Centro di Riferimento Oncologico Aviano (CRO) - IRCCS, Italy Research support: writing, search on the web for experimental drugs in clinical trials.

Elisa Giacomin Hospital Pharmacy Unit, Centro di Riferimento Oncologico Aviano (CRO) - IRCCS, Italy. Research support: writing, search on the web and oncology websites

Paola Molin , Child Neuropsychiatric Unit "Bassa Friulana", Udine University Health Service (ASUFC), Italy. Research support: initial idea, concept: the psychological iimpact of the pandemic scenario on cancer patients; writing,

Liana Dalmas , Hospital Pharmacy Unit, Centro di Riferimento Oncologico Aviano (CRO) - IRCCS, Italy Quality and data control, tables editing

Fabio Puglisi Department of Medicine, University of Udine; Department of Medical Oncology, Centro di Riferimento Oncologico Aviano (CRO) - IRCCS, Italy. Research support: general support, writing the point of view of the clinical oncologist and insights needed.

Running title : Caring cancer patients in the pandemic era: insights

Disclaimer: NONE

SUPPORT \& FUNDING: This research did not receive any specific grant from funding agencies in the public, commercial, or not-for-profit sectors.

\section{Refs of Corresponding Author:}

Paolo Baldo

Hospital Pharmacy Unit, Centro di Riferimento Oncologico Aviano (CRO) - IRCCS, Italy.

Via F. Gallini, 2 -33081 Aviano (PN) Italy,

Tel +39-0434-659221

Mobile: +39-340-5744979

Mail 1: pbaldo@cro.it

Mail 2:paolo.baldo62@gmail.com

After the declaration made on 11.3 .2020 by World health organization, concerning the characterization of SARS-CoV2 infection ${ }^{1}$ as a global pandemic, a colossal amount of scientific studies and research have been published in short times.

To date, published papers are mainly viewpoints or research letters ${ }^{2,3,4}$ but recently, attention to the problem was drawn by Van de Haar et al. on Nature Medicine journal ${ }^{5}$. First of all, the problem of the psychological impact caused by fear of COVID-19 infection on cancer patients should not be underestimated. The devastating threat of a pandemic looming over the social community risks blocking hospital efficiency and care. What are the most critical situations encountered by cancer patients in this scenario?

Therapeutic continuity or postponing therapies. In order to reduce the flow of people, and therefore the risk of spreading the infection, in Cancer Centers the clinical activities has been reduced to the essentials. The reason for these measures lies, first of all, in the particular condition of cancer patients who, due to potential immunodepression, could be more vulnerable to the contraction of infection. Furthermore, as documented for other comorbidities, the co-existence of infection and tumor disease makes patients more prone to complications and exposes them to a higher risk of death. 
But who decides and how do you establish what is essential? The complexity of the therapeutic choices in oncology, linked to the different variables that guide the treatments of the different tumor types, makes it difficult for the same specialists to define the priority criteria. Furthermore, the uncertainty as to how long the adoption of rigid measures for the prevention of infection spread can further complicate decisions on whether or not to postpone or omit certain therapies. The first report on Chinese cancer patients suggested postponing adjuvant therapies or elective surgery ${ }^{6}$.

However, other insights are needed:

1. Definition of the expected benefit of the treatment and of the possible damage for the patient in case of delay in the administration (the reduction of efficacy linked to the lack of respect of the dose density is documented for few clinical situations);

2. The presence of the tumor on site (eg early disease treated with a neoadjuvant approach or advanced disease) and its biomolecular characteristics (eg. proliferative index, unfavorable prognostic factors, etc.) may require more timely treatment;

3. In advanced disease, the treatment line could be a criterion for deciding whether or not to postpone administrations, but this criterion alone could be insufficient;

4. Supportive care and palliative care often require hospital management and, even if they can be delivered at home, they may be subject to delays due to the lack of health workers.

Therefore, although they may appear rigid, measures that limit hospital admissions are necessary to protect both patients and healthcare professionals from the risk of infection. At the same time, these measures generate a state of isolation for the patient who is facing the diagnostic-therapeutic path without the support of family members or caregivers.

Availability of hospital beds, and information overload by the mass media. Right now, everyone, mainly in Europe, is exposed to a media bombing about the Covid-19 pandemic. Even cancer patients are not excluded, and it can be very impressive the hypothesis that health systems may have to choose, next days, whether to reserve care, for example, to a 70-year-old adult suffering from severe COVID-19 infection but without comorbidities, who therefore has a more likely to be cured, or an adult who has other concomitant serious diseases, such as cancer. This is an issue posed by logistical and economic reasons, but that implies profound ethical considerations by health organizations.

Discomfort in having to take interviews and triage. Continuing to refer to the restrictions imposed by the Italian government, which are even stricter starting from $12 / 3$, these require that every person entering hospital institutions carry out a synthetic triage declaring the absence of symptoms, i.e. the presence of rhinitis, fever, cough, difficulty breathing and a brief interview about the personal recent history of social contacts or movements. It is essential to remember that the cancer patient can occasionally be immunocompromised, for example with the presence of neutropenic fever. This can be confusing for healthcare professionals who perform triage, but also confusing if there is a potential hiding of the COVID-19 infection. Certainly, in any case, the moment of the interview or triage represents an "appointment" with fear and anxiety for the cancer patient who sees the guarantee of the chemotherapy cycle or the follow-up visit questioned.

Indirect consequences: emotional and psychological costs on the families of cancer patients. In addition, primarily in Italy, government restrictions impose an almost total absence of movement outside one's home, at least until the end of March. The indirect consequences are social isolation due to possible quarantine ${ }^{7}$, reduction of assistance and closeness of the family members to cancer patients, who however cannot and must not feel "alone" to face the most critical phase of their life: coping with cancer, let's not forget it.

The position of Scientific Societies of Oncology and Haematology. In the present, unpredictable scenario, which has found the medical world unprepared, the position of representative Scientific society in oncology field may be differently oriented. Some scientific societies are mainly oriented to give information to associates that all events, conferences, meetings or training courses are postponed or canceled; others include in the 
website homepage special sections about the COVID-2019 pandemic emergency, expressing the Society's point of view and behavioral indications.

For example, AIOM , the Italian Association of Medical Oncology (www.aiom.it), is informing patients in the website homepage that it might be necessary to defer part of the planned activity, access to pharmacologic treatment and to postpone follow-up, non urgent visits. The advice is also to evaluate and discuss case by case any access to the treatment, based on the relationship between the risks (for the individual and for the community) related to hospital access and the benefits expected from the treatment itself. On March 13, 2020, AIOM released the document: "Infectious risk from Coronavirus COVID 19: indications for Oncology". In this document, AIOM considers it appropriate to postpone the outpatient activities of follow-up for diseasefree patients (e.g. follow-up at 6-12 months) partially, providing for a telephone call and conversation. At the same time, to avoid overcrowding of waiting rooms, it is advised that access of the accompanying persons in the rooms where therapy is administered or within surgeries, will be limited to one for each patient. \sout. $\boldsymbol{A S C O}$, the American Society of Clinical Oncology (https://www.asco.org/asco-coronavirus-information) is presenting in the website homepage the "ASCO Coronavirus resources", consisting in essential links for information about the new Coronavirus disease for health professionals involved in cancer care; the society has also developed a guidance for patients, based on questions received from ASCO members and the internal COVID-19 Clinical Questions Advisory Group.

ESMO, the European Society for Medical Oncology, (https://www.esmo.org) proposes rich, informative material for oncology professionals and cancer patients; in parallel, ESMO has launched a collaborative project, the ESMO-CoCARE Registry, to share data and information among healthcare professionals about treatment approaches on cancer patients who are suspected or confirmed to have the novel coronavirus disease (COVID-19). The aim is to understand better the unique effects of COVID-19 on patients with cancer and cancer survivors. By pooling real-world data with updated information coming from clinical and pharmacological research, ESMO hopes to assist cancer patients at best.

LLS - Leukemia \& Lymphoma Society (https://www.lls.org/blog/4-things-cancer-patients-need-to-knowabout-the-coronavirus) is perhaps the more closest to the spirit of this paper, declaring in its homepage that one of its highest priorities is "Helping Blood Cancer Patients Adjust to the New Normal" telling about the new COVID-2019 pandemic. LLS priority is the well-being of the patients - especially as many blood cancer patients are immunocompromised, suggesting the " 4 things cancer patients need to know about the Coronavirus disease".

It is of paramount importance that all the scientific societies indicate patients to follow the directives of the national regulatory authorities and the other competent authorities, for example, the Centers for Disease Control and Prevention (CDC) or World Health Organization (WHO) guidelines, in order to protect the health of all of us and the general population ${ }^{8}$. All healthcare professionals can have access to electronic directories reporting links to the clinical reference guidelines ${ }^{9,10,11}$. Finally, in the homepage of clinicaltrials.gov (www.clinicaltrials.gov) ${ }^{12}$, a direct filter strategy available links us directly to all the clinical trials activated for COVID-19 research, a list that on 13.5.2020 reached the number of 1.450 registered studies in the world.

In conclusion, while the various countries are waiting to see a drop in the number of infections and deaths caused by COVID-2019, a great effort by all health professionals is needed to do their best to help in this new healthcare scenario. Healthcare professionals dealing with cancer patients must also avoid any form of distraction and concentrate their efforts on communication to transmit messages of trust and hope, especially needed in these critical times.

\section{CONFLICT OF INTEREST STATEMENT}

\section{None to declare}

SUPPORT \& FUNDING: This research did not receive any specific grant from funding agencies in the public, commercial, or not-for-profit sectors. 


\section{REFERENCES}

1. Wu Y, Ho W, Huang Y, et al. SARS-CoV-2 is an appropriate name for the new coronavirus. The Lancet. 2020;395(10228):949-950.

2. Xia Y, Jin R, Zhao J, Li W, Shen H. Risk of COVID-19 for cancer patients. The Lancet Oncol. 2020;21(4):E180. doi:https://doi.org/10.1016/S1470-2045(20)30150-9

3. Wang H, Zhang L. Risk of COVID-19 for patients with cancer. The lancet Oncol . 2020;21(4):E181. doi:https://doi.org/10.1016/S1470-2045(20)30149-2

4. Cortiula F, Pettke A, Bartoletti M, Puglisi F, Helleday T. Managing COVID-19 in the oncology clinic and avoiding the distraction effect.Annals of Oncology . 2020;5:553-5. doi:https://doi.org/10.1016/j.annonc.2020.03.286.

5. Van de Haar J, Hoes LR, Coles CE. et al. Caring for patients with cancer in the COVID-19 era. Nat Med. 2020;26:665-671.

6. Liang W, Guan W, Chen R, et al. Cancer patients in SARS-CoV-2 infection: a nationwide analysis in China. The Lancet Oncol . 2020;21(3):335-337.

7. Brooks SK, Webster RK, Smith LE, et al. The psychological impact of quarantine and how to reduce it: rapid review of the evidence. The Lancet . 2020;395(10227):912-920.

8. WHO Guideline: Clinical management of severe acute respiratory infection when novel coronavirus (nCoV) infection is suspected. Interim guidance. Available at website:https://www.who.int/publications-detail/clinical-management-of-severe-acute-respiratoryinfection-when-novel-coronavirus-(ncov)-infection-is-suspected(accessed 23.3.2020).

9. Medscape Directory. COVID-19 Clinical Guidelines . Available at webpagehttps://www.medscape.com/index/list_13405_0(last accessed 13.5.2020).

10. Uptodate Covid-19 guidelines directory. Available at webpage:https://www.uptodate.com/contents/societyguideline-links-coronavirus-disease-2019-covid-19-international-and-government-guidelines-forgeneral-care(last accessed 13.5.2020).

11. BMJ Best Practice Guidelines. Coronavirus Disease 2019 . Available at webpage:https://bestpractice.bmj.com/topics/en-gb/3000168/guidelines(last accessed 13.5.2020).

12. ClinicalTrials.gov. Registry and results database of publicly and privately supported research studies in the U.S.A and around the world. Filter to retrieve studies for Sars-CoV-2 infection. Available at webpage:https://clinicaltrials.gov/ct2/results? cond=COVID-19(last accessed 14.5.2020). 